\title{
Upgrade of the ATLAS Tile Calorimeter for the High Luminosity LHC
}

\author{
Krystsina Petukhova*, on behalf of the ATLAS Tile Calorimeter System \\ Charles University, Prague, Czech republic \\ E-mail: krystsina.petukhova@cern.ch
}

The Tile Calorimeter is the hadronic calorimeter covering the central region of the ATLAS experiment. TileCal is a sampling calorimeter with steel as absorber and scintillators as active medium. The scintillators are read out by wavelength shifting fibers coupled to photomultiplier tubes (PMTs). The analogue signals from the PMTs are amplified, shaped, digitized by sampling the signal every $25 \mathrm{~ns}$, and stored on detector until a trigger decision is received. The HighLuminosity phase of LHC (HL-LHC) expected to begin in year 2026 requires new electronics to meet the requirements of a $1 \mathrm{MHz}$ trigger, higher ambient radiation, and better performance under high pileup. Both the on- and off-detector TileCal electronics will be replaced during the shutdown of 2024-2025. PMT signals from every TileCal cell will be digitized and sent directly to the back-end electronics, where the signals are reconstructed, stored, and sent to the first level of trigger at a rate of $40 \mathrm{MHz}$. This will provide more precise calorimeter signals used by the trigger system and will allow the development of more complex trigger algorithms. Changes to the electronics will also contribute to the data integrity and reliability of the system. Results are presented from a prototype of the new electronics ('demonstrator') that was inserted in a TileCal module and tested in CERN H8 beamline with electrons, muons, and hadrons. The demonstrator is undergoing extensive testing and will be inserted in the ATLAS detector during the current shutdown.

XXIX International Symposium on Lepton Photon Interactions at High Energies - LeptonPhoton2019 August 5-10, 2019

Toronto, Canada

${ }^{*}$ Speaker. 


\section{Introduction}

The hadronic Tile Calorimeter covers the central region of the ATLAS experiment [1] at the LHC [2]. It measures the energy of hadrons, jets, $\tau$-leptons, and missing transverse energy. The High-Luminosity LHC (HL-LHC) will operate with the instantaneous luminosity of 5-10 times larger than the nominal LHC value of $10^{34} \mathrm{~cm}^{-2} \mathrm{~s}^{-1}$. The TileCal will require a new electronics to provide a low-latency, high-frequency $(40 \mathrm{MHz}$ ), fully digital input for ATLAS trigger system. All the components need to withstand higher ambient radiation (2-24 Gy for $4 \mathrm{ab}^{-1}$ ) and the high luminosity environment ( $\sim 200$ collisions per bunch crossing). The upgrade provides an increased data throughput with high-performance large-width readout links (40 Tbps for the entire detector readout [3]). The system reliability is assured by the power supply, data links redundancy, and the readout modularity. The calibration infrastructure will be improved with respect to the LHC Run 1 experience. About $10 \%$ of the most exposed PMTs will be replaced due to radiation and time aging.

\section{The TileCal electronics in the HL-LHC upgrade}

The TileCal is composed of 256 wedge-shaped modules, each housing a Superdrawer (SD). A SD is divided into 4 Minidrawers (MDs), each with an independent readout and a separate power supply (Figure 1b). A MD hosts 12 PMTs and 12 Front-End Boards (FEB) named FENICS (Front End board for the New Infrastructure with Calibration and signal Shaping, Figure 1a). The Mainboard (MB) shapes, amplifies (two gains), and digitizes the input with 12-bit ADCs [4] covering a 17-bit dynamic range (0-800 pC). The Daughterboard (DB) transfers the high-speed output to the back-end, distributes LHC clock settings, and connects the the on- and off-detector electronics [5]. The Tile PPr (Preprocessor) buffers data from all the MDs in the off-detector pipelines, evaluates signal and calculates trigger objects at the LHC rate, and distributes the sampling clock and detector control information. The Trigger and Data Acquisition interface (TDAQi) sends accepted data to the FELIX (Front End LInkeXchange). The upgraded High Voltage (HV) Power Supply has better temperature and voltage stability, along with remote or local control. The Low Voltage Power Supply (LVPS) has lower noise and point-of-load regulators.

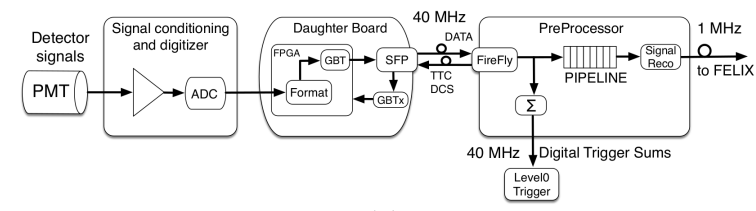

(a)

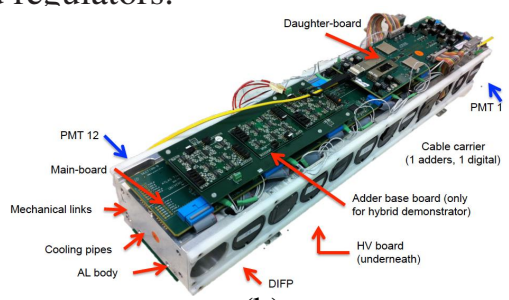

(b)

Figure 1: The layout of the readout and electronics (a) [6]. The TileCal minidrawer (b) [7].

\section{Beam and radiation tests}

The TileCal Demonstrator is a hybrid drawer with the upgraded electronics prototype and the legacy TDAQ, detector control and calibration systems. The Hybrid Demonstrator underwent 7 test beam campaigns (electrons, muons, hadrons) at the CERN SPS on the H8 beamline facility in 20152018. The test beam results show good performance of the new electronics. The measurements are 
aligned with the results obtained with the legacy system [8]. The dependence of the cell response to muons on the impact point $\phi$ has the expected U-shape (Figure 2a). The response is linear over a wide range of incident particles energy (Figure $2 b$ ). The low-energy signal is well separated from noise (Figure 2c). The components underwent continuous radiation tolerance tests which showed no destructive errors or latch-ups and verified the LVPS voltage stability.

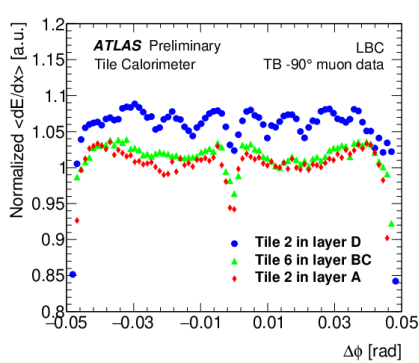

(a)

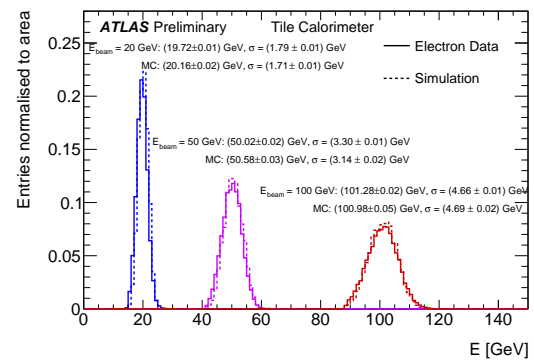

(b)

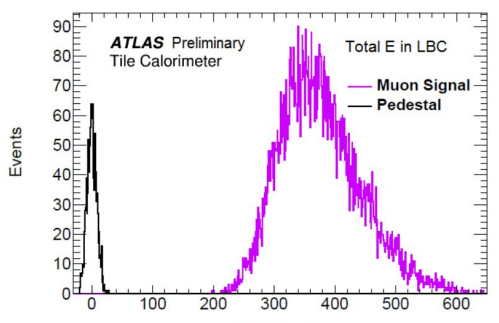

(c)

Figure 2: The cell response dependence on $\phi$ of the muon impact point (a) [8]. The energy deposited during electron beams (b) [8]. The separation of low-energy signal and noise (c) [8].

\section{Outlook}

In July 2019 (during Long Shutdown 2), a prototype of the TileCal was inserted in the ATLAS detector. Functionality of the prototype will be verified. It will be eventually evaluated for datataking in Run 3. The prototype includes the HL-LHC electronics, the remote HV control and the 3-in-1 FEB, which is a predecessor of the FENICS including analog trigger output for the present ATLAS trigger system. The Tile PPr demonstrator (1/8 of the full-size HL-LHC PPr) will provide data to the current DAQ and transmit triggered events to the FELIX. The main elements are about to enter pre-production. All the on- and off-detector TileCal electronics will be replaced in 2024-2026 for the HL-LHC era.

\section{Acknowledgments}

The financial support of the Visegrad fund and the grant LTT 17018 of the Ministry of Education, Youth and Sport of the Czech Republic is acknowledged. Testbeam activities were supported by the Advanced European Infrastructures for Detectors at Accelerators (AIDA 2020).

\section{References}

[1] ATLAS Collaboration, JINST 3 (2008) S08003.

[2] L. Evans and P. Bryant, LHC Machine, JINST 3 (2008) S08001.

[3] F. Carrió, A. Valero, on behalf of the ATLAS Tile Calorimeter System, IEEE 661014.

[4] F. Tang, on behalf of the ATLAS Tile Calorimeter System, Springer Proc. Phys. 213 (2018) 22.

[5] E. Valdes Santurio, S. Silverstein, C. Bohm, PoS 340 (2018).

[6] https://twiki.cern.ch/twiki/pub/AtlasPublic/ApprovedPlotsTileUpgrades.

[7] F. Scuri, on behalf of the ATLAS Tile Calorimeter System, Journal of Physics: Conference Series 1162 (2019) 012017.

[8] https://twiki.cern.ch/twiki/bin/view/AtlasPublic/ApprovedPlotsTileTestBeamResults. 